\title{
Numerical Simulation of Unsteady Channel Flow with a Moving Indentation
}

\author{
$C R$ Sonawane ${ }^{1, \mathrm{a},} Y$ B More ${ }^{1}$, and Anand Pandey ${ }^{1}$
}

${ }^{1}$ Mechanical Engineering Department, Symbiosis Institute of Technology, Symbiosis International (Deemed University), Pune, India.

\begin{abstract}
The FSI problem - unsteady channel flow with a moving indentation problem, which represents flow features of oscillating stenosis of a blood vessel, is numerically simulated. The flow inside the channel with moving boundary results in transient and complex flow phenomena mainly due to the interaction between the moving boundary and the flowing fluid. In this paper, an accurate Harten Lax and van Leer with contact for artificial compressibility Riemann solver have been used for flow computation. The Riemann solver is modified to incorporate Arbitrarily Lagrangian-Eulerian (ALE) formulation in order to take care of mesh movement in the computation, where radial basis function is used for dynamically moving the mesh. Higher order accuracy over unstructured meshes is achieved using quadratic solution reconstruction based on solution dependent weighted least squares (SDWLS). The present numerical scheme is validated here and the numerical results are found to agree with experimental results reported in literature.
\end{abstract}

\section{Introduction}

Incompressible flows over moving boundaries are encountered in many practical situations. The main feature of these flows is their unsteadiness with respect to shape of the boundaries and corresponding flow patterns. One such example is the flow over a moving indentation inside a channel [1], which represents flow features of oscillating stenosis of a blood vessel. The flow inside the channel with moving boundary results in transient and complex flow phenomena mainly due to the interaction between the moving boundary and the flowing fluid.

Pedley et al. [1] had carried out the experimental investigation on the moving channel at various reynolds number with chanel flutuation (related with Strouhal number). They observe the generation of vortices producing from the top fixed wall as well as bottom moving wall. Ralph et al. [2] simulated the viscous and inviscid flows in a channel with a moving indentations and confirms that the continues generation of vortex which ultimetely related to the pressure variation across the channel. Zhao et al. [3] also simulated the the channel flow by using higher order accurate method applied over unstructured mesh using artifical compressibility method. Mandal et al. [4] had attempted simulation using artificial compressibility method. The reults of all mentioned literatures are found to be matching well, however they were also indicating a scope to capture the flow physics in a better manner.

In this paper, the FSI problem - unsteady channel flow with a moving indentation problem is studied using a numerical method. An accurate Harten Lax and van Leer with contact for artificial compressibility (HLLC-AC) Riemann solver [4 - 6] developed for solving incompressible flows in artificial compressibility formulation have been used for flow computation. The Riemann solver is modified to incorporate Arbitrarily Lagrangian-Eulerian (ALE) [7] formulation in order to take care of mesh movement in the computation, where radial basis function [8] is used for dynamically moving the mesh. Higher order accuracy is achieved using quadratic solution reconstruction based on solution dependent weighted least squares (SDWLS) [9]. The results obtained by the present method is validated against the results reported in the literature.

\section{Arbitrarily Lagrangian-Eulerian (ALE) formulation}

The integral form of 2D unsteady incompressible NavierStokes equations in artificial compressibility [10] formulation, with Dual time steeping (DTS) approach considered for time accurate solution, written in Arbitrarily Lagrangian-Eulerian (ALE) [7] formulation for mesh movement as,

$$
\begin{aligned}
& \iint_{\Omega} \frac{\partial \mathrm{W}}{\partial \tau} \mathrm{dxdy}+\mathrm{I}^{\mathrm{M}} \iint_{\Omega} \frac{\partial \mathrm{W}}{\partial \mathrm{t}} \mathrm{dxdy}+\Theta^{\mathrm{M}} \oint_{\mathrm{A}}\left[\left(\mathrm{E}^{\mathrm{c}}+\right.\right. \\
& \left.\left.\mathrm{E}^{\mathrm{v}}\right) \mathrm{n}_{\mathrm{x}}+\left(\mathrm{F}^{\mathrm{c}}+\mathrm{F}^{\mathrm{v}}\right) \mathrm{n}_{\mathrm{y}}\right] \mathrm{dA}=\iint_{\Omega} \mathrm{S}_{0} \mathrm{dxdy}
\end{aligned}
$$

\footnotetext{
* Corresponding author: author@e-mail.org
} 


$$
\begin{aligned}
& W=\left\{\begin{array}{c}
p / \rho \\
u \\
\mathrm{v}
\end{array}\right\} ; E^{c}=\left\{\begin{array}{c}
U \\
U u+p / \rho\} \\
\mathrm{Uv}
\end{array}\right\} ; \\
& G^{c}=\left\{\begin{array}{c}
V \\
u V \\
v V+{ }^{p} / \rho
\end{array}\right\} ; E^{v}=\left\{\begin{array}{c}
0 \\
\sigma_{x x} \\
\sigma_{x y}
\end{array}\right\} ; G^{v}=\left\{\begin{array}{c}
0 \\
\sigma_{y x} \\
\sigma_{y y}
\end{array}\right\} ; \\
& I^{M}=\left\{\begin{array}{lll}
1 & 0 & 0 \\
0 & 1 & 0 \\
0 & 0 & 1
\end{array}\right\} ; \Theta^{M}=\left\{\begin{array}{lll}
\beta^{2} & 0 & 0 \\
0 & 1 & 0 \\
0 & 0 & 1
\end{array}\right\} ; S_{0}=\left\{\begin{array}{c}
0 \\
f_{e, x} \\
f_{e, y}
\end{array}\right\}
\end{aligned}
$$

where, $U=u-x_{t}$ and $V=\mathrm{v}-y_{t}$ are the convective velocities in referential frame with $x_{t}$ and $y_{t}$ are the velocities of the moving grid in $\mathrm{X}$ and $\mathrm{Y}$ directions respectively. Note that, equation (1) does not exhibit any physical meaning until pseudo time steady state, i.e. $\left(\frac{\partial \mathrm{p}}{\partial \tau}=\frac{\partial \mathrm{u}}{\partial \tau}=\frac{\partial \mathrm{v}}{\partial \tau} \cong 0\right)$ is reached. As the pseudo-steady state is reached, the equations are identical to the original unsteady incompressible Navier-Stokes equations in ALE form.

Now splitting the convective fluxes $\left(E^{c}\right.$ and $\left.G^{c}\right)$ of equation (1) into stationary reference flux and ale flux part as,

$$
\begin{aligned}
& \iint_{\Omega} \frac{\partial \mathrm{W}}{\partial \tau} \mathrm{dxdy}+\mathrm{I}^{\mathrm{M}} \iint_{\Omega} \frac{\partial \mathrm{W}}{\partial \mathrm{t}} \mathrm{dxdy}+\Theta^{\mathrm{M}} \oint_{\mathrm{A}}\left[\left(\mathrm{E}_{\mathrm{St}}^{\mathrm{c}}+\right.\right. \\
& \left.\left.\mathrm{E}^{\mathrm{v}}\right) \mathrm{n}_{\mathrm{x}}+\left(\mathrm{F}_{\mathrm{st}}^{\mathrm{c}}+\mathrm{F}^{\mathrm{v}}\right) \mathrm{n}_{\mathrm{y}}\right] \mathrm{dA}-\oint_{\mathrm{A}}\left[\left(\mathrm{E}_{\mathrm{ale}}^{\mathrm{c}}\right) \mathrm{n}_{\mathrm{x}}+\right. \\
& \left.\left(\mathrm{F}_{\mathrm{ale}}^{\mathrm{c}}\right) \mathrm{n}_{\mathrm{y}}\right] \mathrm{dA}=\iint_{\Omega} \mathrm{S}_{0} \mathrm{dxdy}
\end{aligned}
$$

Now equation (4) can be discretized in a very similar manner to that for unsteady Navier-Stokes equation for stationary boundary problem [4 - 6]. The additional effort need to be added for ale flux vector. This additional term, ale flux, is nothing but the volumetric increment along the face and can be evaluated by considering the Geometric Conservations Law (GCL) [8].

The radial basis function [11]: Thin-Plate Spline (TPS) with global support is used for mesh movement. The Thin Plate Spline with global support generates meshes of high quality after deformation along with the computational efficiency.

The fluxes at cell interface, that is, the stationary reference convective fluxes are evaluated using the Harten Lax and van Leer with contact for artificial compressibility (HLLC-AC) [4 - 6] Riemann solver. Higher order accuracy is achieved using quadratic solution reconstruction, where interface values are reconstructed based on solution dependent weighted least squares (SDWLS) [9]. For viscous fluxes, a central differencing method based on Green-Gauss approach is used.

\section{Channel flow with a moving indentation}

The geometry and dynamics of the moving indentation channel chosen here is identical to the experimental work of Pedley and Stephanoff [1]. The aspect ratio of the channel is 10 , and features of the flow are observed to be uniform in the span-wise direction over most of the flow cycle, so that a two-dimensional simulation is appropriate. The schematic of flow problem is shown in Fig. 1.

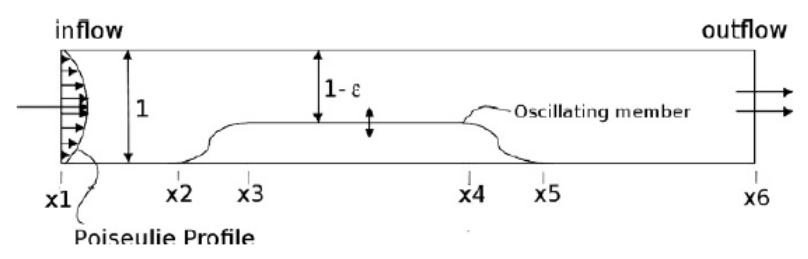

Figure 1: Schematic of the problem setup.

The shape of indentation varies as a function of space and time $f(x, t)$ as,

$$
f(x, t)=g(x) \cdot h(t)
$$

where $h(t)$ is given as,

$$
\begin{aligned}
& h(t)= \frac{1}{2}[1-\cos 2 \pi t / T], \quad t / T \in[0,1] \\
& g(x)=\left\{\begin{array}{ll}
0 & \text { for } x_{1}<x \leqslant x_{2} \\
\frac{1}{2} \epsilon\left[1+\tanh \beta_{d y n}\left(x-x_{a}\right)\right] & \text { for } x_{2}<x \leqslant x_{3} \\
\epsilon & \text { for } x_{3}<x \leqslant x_{4} \\
\frac{1}{2} \epsilon\left[1+\tanh \beta_{d y n}\right] & \text { for } x_{5}<x \leqslant x_{5} \\
\epsilon & \text { for } x_{5}<x \leqslant x_{6}
\end{array}\right\}
\end{aligned}
$$

The parameters, $\mathrm{x} 1=-13.75, \mathrm{x} 2=-11.75, \mathrm{x} 3=-9.25, \mathrm{x} 4$ $=-1.25, \mathrm{x} 5=1.25, \mathrm{x} 6=13.25, \varepsilon=0.38$, and $\beta$ dyn $=4.14$, similar to those of Zhao and Forhad [3] have been chosen.

No slip boundary condition is applied on the walls, which is specified by imposing $(\mathrm{u}, \mathrm{v})$ wall $=(0,0)$ on static walls, and $(\mathrm{u}, \mathrm{v})$ wall $=(\mathrm{xt}$, yt) wall on moving wall. At inlet, a Poiseuille velocity profile is specified as boundary condition. At the outlet, back pressure, $\mathrm{pb}$ is specified and velocities are extrapolated. The inlet pressure is computed from the interior.

\section{Result and discussion}

A quantitative comparison between the present results and literature results $[1-3]$ in terms of time evolution of shed vortices 'B', 'C', 'D' are presented graphically in Fig. 2, which are found to be in good agreement. The 
discrepancies seen here are within the range of experimental scatter. The discrepancies are also due to expected lower accuracy associated with finding locations of vortices, which are based on derived quantity (vorticity) involving differentiation.

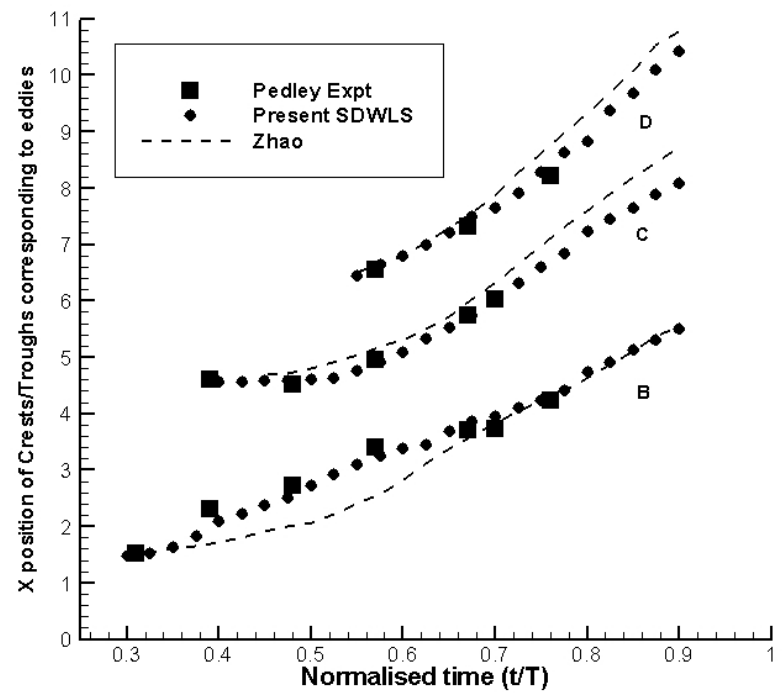

Fig 7: Time evolutions of shed vortices 'B', 'C', and ' $D$ '

\section{Conclusion}

An accurate Harten Lax and van Leer with contact for artificial compressibility (HLLC-AC) Riemann solver with arbitrarily Lagrangian-Eulerian (ALE) formulation has been developed and used for computing unsteady channel flow with a moving indentation. The results obtained by the present solver matches well with that reported in the literature.

\section{Acknowledgments}

This work is supported by Science and Engineering Research Board (SERB) - Department of Science and Technology (DST), Government of India (ECR/2017/000476).

\section{References}

1. Pedley T J, Stephanoff K D., J Fluid Mech 1985;160:337-67.

2. Ralph M E, Pedley T J., J Fluid Mech, 1989; 209:543-66.

3. Zhao Y, Forhad A. , Appl MechEng 2003;192:443966.

4. J C Mandal, C R Sonawane, A S Iyer and S J GosaviInamdar, Computers \& Fluids, 46 (2011) 348352

5. C R Sonawane and J C Mandal, International Journal of Numerical Methods for Heat and Fluid Flow,. Volume 23, Issue 1, 2013
6. C R Sonawane and J C Mandal, Journal of Heat Transfer Engineering, Vol 35, Issue 11/12 July/August 2014.

7. Cook J L, Hirt C W, Amsden A A, Journal of Computational Physics, 14, Issue 3:227-253, March 1974

8. H Bijl, A de Boer, M S van der Schoot, Computers and Structures, 85:784-795, 2007.

9. C R Sonawane, J C Mandal and S. P. Roa, J. Inst. Eng. India Ser. C (2017). https://doi.org/10.1007/s40032-017-0390-x

10. A J Chorin, Math Comput, 22, 745-762, 1968.

11. Lombard C K, Thomas P D, AIAA Journal, 17:1030 1037, 1979.

12. E. F. Toro, M. Spruce, W. Speares, Shock Waves, 4, 25-34, 1994.

13. J. C. Mandal, S. P. Rao, Computers and Fluids, 44, 23-31, 2011.

14. C. F. V. Loan, G. H. Golub., 3rd ed. The Johns Hopkins University Press, 1996.

15. C. Bischof, S. Blackford, J. Demmel, J. Dongarra, E. Anderson, Z. Bai, Society for Industrial and Applied Mathematics, 1999. 